\title{
A Singleton Type-1 Fuzzy Logic Controller for On-Line Error Compensation During Robotic Welding
}

\author{
I. Davila-Rios, I. Lopez-Juarez, G.M. Mendez, \\ R. Osorio-Comparan, G. Lefranc, C. Cubillos
}

Ignacio Davila

COMIMSA - Posgrado Interinstitucional en

Ciencia y Tecnologia (PICYT)

Ciencia y Tecnologia No 790 Col. Saltillo 400, C.P. 25290 Saltillo, Coahuila. Mexico.

idavila@comimsa.com

\section{Ismael Lopez-Juarez*}

Robotics and Advanced Manufacturing Group

Centro de Investigacion y de Estudios

Avanzados del IPN (CINVESTAV)

Av. Ind. Metalurgica 1062, P. Ind.

Saltillo-Ramos Arizpe, C.P. 25900 Ramos

Arizpe, Coahuila, Mexico

*Corresponding author:

ismael.lopez@cinvestav.mx

\section{Gerardo Maximiliano Mendez}

Instituto Tecnologico de Nuevo Leon (ITNL)

Av. Eloy Cavazos 2001 Col. Tolteca, C.P. 67175

Guadalupe, Nuevo Leon, Mexico

gerardo.maximiliano.mendez@gmail.com

\author{
Roman Osorio-Comparan \\ Instituto de Investigaciones en Matematicas \\ Aplicadas y Sistemas \\ Universidad Nacional Autonoma de Mexico \\ (UNAM) \\ Circuito Escolar S/N, Ciudad Universitaria, \\ Coyoacán, C.P. 04510, Mexico City \\ roman@unam.mx
}

\section{Gaston Lefranc}

Escuela de Ingenieria Electrica

Pontificia Universidad Catolica de Valparaiso Avda Brasil 2950, Valparaíso, Chile 2430000 gaston.lefranc@pucv.cl

\section{Claudio Cubillos}

Escuela Ingenieria Informatica

Pontificia Universidad Catolica de Valparaiso Avda Brasil 2950, Valparaíso, Chile 2430000 claudio.cubillos@pucv.cl

\begin{abstract}
During robot welding operations in the manufacturing industry there is a need to modify on-line the welding path due to a mismatch in the position of the components to be welded. These positioning errors are due to multiple factors such as ageing of the components in the conveyor system, clamp fixtures, disturbances, etc. Therefore, robot reprogramming is needed which requires a stop in the production line and consequently an increment in production costs. This article is an extension of [1] $]^{a}$ and presents an alternative solution to this problem that involves the use of structured lighting using a low-cost laser beam, a CMOS camera and a Gaussian singleton fuzzy logic controller. To validate the proposed control system, a robotic cell was designed using an industrial KUKA KR16 robot for welding metallic plates. The method was evaluated experimentally under lateral and vertical positioning errors.

Keywords: Gas Metal Arc Welding (GMAW), industrial robotics, artificial vision, robot path control, fuzzy logic.

\footnotetext{
${ }^{a}$ Reprinted (partial) and extended, with permission based on License Number 3947080516854 [2016] CCIEEE, from "Computers Communications and Control (ICCCC), 2016 6th International Conference on".
}

\section{Introduction}

The welding process establishes an electrical arc between a continuously fed electrode and the weld pool; which is protected by a gas administered externally, hence its name Gas Metal Arc Welding (GMAW) or commonly known as Metal Inert Gas (MIG). During the process, the molten electrode is transferred to the workpiece through the electric arc and serves as the filler metal (weld bead) which is deposited accurately by and automated mechanism, e.g. by 
an industrial robot. During robot welding manufacturing operations, misalignments are likely to occur. There are several reasons; it can be due to disturbances, positioning sensing errors, ageing of welding fixtures and in general errors in positioning and conveying mechanisms. It is a common practice to make some robot reprogramming to solve the problem that requires the stop of the production line with an increment in production costs. There are currently commercial solutions that can be adapted to solve these common errors, however the costs can be very high, and in the order of the price of the industrial robot itself, which may not be affordable for some small companies. In this article, we present an alternative solution to this problem that involves the use of structured lighting employing a low-cost laser beam, a CMOS camera and a PC-based fuzzy controller.

\section{$1.1 \quad$ Related work and objective}

The work presented in this paper is an extended version of the originally published article in the IJCCC [1]. Similar work on robot control for seam tracking has been presented by Graf et al. [2] who developed a trajectory-based control for seam tracking by modelling the trajectory as a continuous curve in 3D. They showed good results; however, the technique has some drawbacks in cases where the solution does not exist for correcting the orientation of the seam location or if the current robot location is close to the last location. Santti et al. [3] have recently tested high performance processors to extract the dominant line from the segmented data of the trajectory. The system can achieve a line extraction speed of more than $1000 \mathrm{fps}$, which enables real-time visual seam tracking and robot control. Some approaches that only use an optical sensor have been reported. Liu et al. [4] have proposed to use optical filtering and the modification of the camera's exposure time to extract the geometrical profile of the seam. Some other approaches also have appeared in the literature that addresses neuro-fuzzy controllers that can be applied to robot positioning control [5].

The objective of the research presented in this paper is to correct the robot's end effector position online to eliminate the need of reprogramming the robot. In this manner, errors due to disturbances, positioning sensing errors, ageing of welding fixtures and in general errors in positioning and conveying mechanisms are eliminated. Our proposal is based on previous approaches using structure lighting (i.e. laser sensor) and image processing to quantify the misalignment and to react before the actual beam is formed by sending robot commands to reposition the arm robot so that initial offset cannot affect the next piece to be weld in a production line. The performance of the fuzzy controller is verified experimentally.

The paper is structured as follows. In section 2, the test bed is explained. The image processing for detecting the part misalignment is described in Section 3, whereas the design of the controller and its experimental results are explained in sections 4 and 5, respectively. Finally, conclusions, current and envisaged work are given at the end of the paper in Section 6 .

\section{Test bed}

The following equipment composes the test bed: a KUKA robot arm manipulator, GMAW welding station, wire feeder that controls the wire supply to the torch, a PC-based data acquisition system and an inert gas tank as it is depicted in Figure 1

The test bed also includes a video camera and a laser sensor oppositely positioned to each other. By using this configuration, occlusions by the torch itself can be avoided. However, a perspective error occurs which is compensated by a homography matrix when using triangular singleton type-1 (T1) fuzzy logic controller (FLC) and it is directly compensated without the homography when a Gaussian singleton T1 FLC controller is used. With our proposal using the 


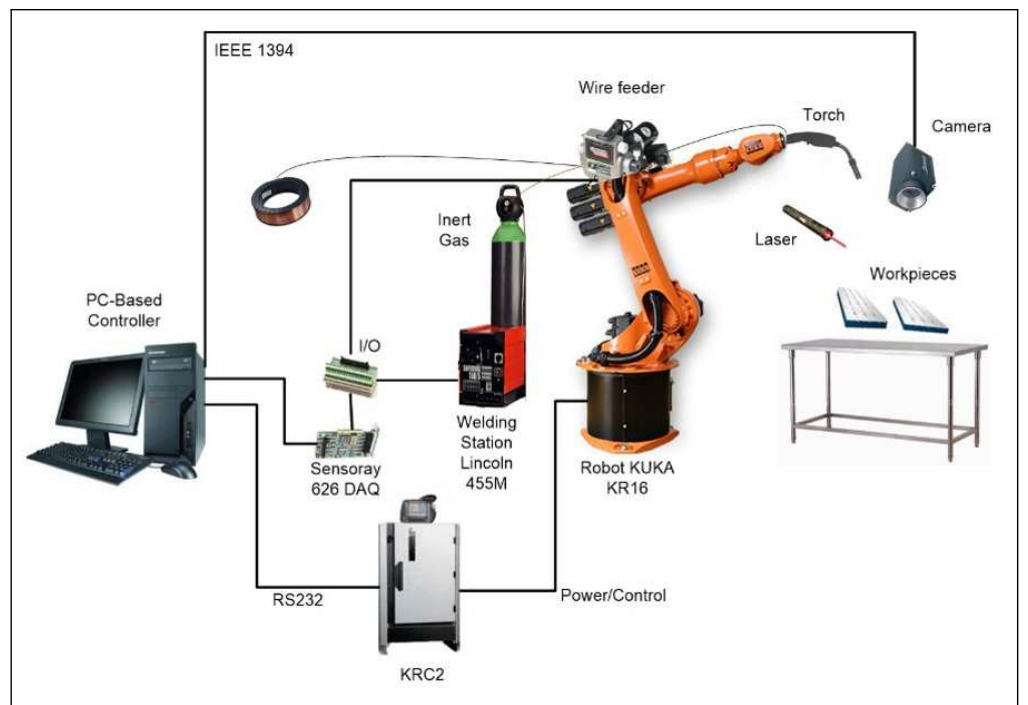

Figure 1: Welding robotised cell.

Gaussian singleton T1 FLC, the use of algorithms for perspective error correction and image processing of high computational costs are avoided.

The welding cell is controlled by a PC-based controller. The computer has an Intel Xeon @ $1.86 \mathrm{GHz}$ processor with $3 \mathrm{~GB}$ RAM that hosts the application interface and which also includes the following functions:

Serial communication. For on-line modification of the welding path. The communications are carried out at low-level between the PC and the KRC2 controller using the 3964 protocol.

Image processing. Filtering and segmentation are carried out using a CMOS Basler A602fc camera as input device with a spatial resolution of $656 \times 490$ pixels.

Voltage and current modification. The arch current and voltage is modified by DAQ Sensoray 626 using its I/O port. Different I/O signals are used to control the on/off of the welding station, the wire feeding system (including the open/close state of the gas valve), the laser sensor on/off condition, the welding travel speed and the distance from the tip of the torch to the workpiece.

The robot manipulator is used in slave mode. During the operations, a robot positioning program is run in the $\mathrm{KRC} 2$ controller that continuously search for motion commands from the PC controller in order to start an incremental motion of the robot arm. This program also controls the selection between tool and world coordinates, and the speed and motion step size during incremental motions. The positioning fuzzy controller resides in the PC controller sending the path modification commands to the robot controller during welding operations.

\section{$3 \quad$ Image processing}

During image processing some distortion can occur due to the alignment of the camera with respect to the image plane as it is shown in Figure 2.

A homography relates two images in perspective, where plane points from one scene are related to the second image. This relationship is valid if the scene is flat or the displacement small [6] as it is illustrated in Figure 3.

In this manner the homography helps to eliminate the perspective from one image and also 


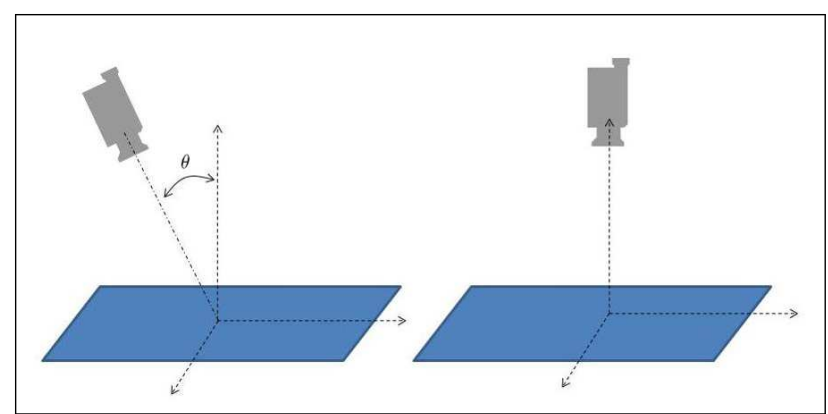

Figure 2: Camera alignment

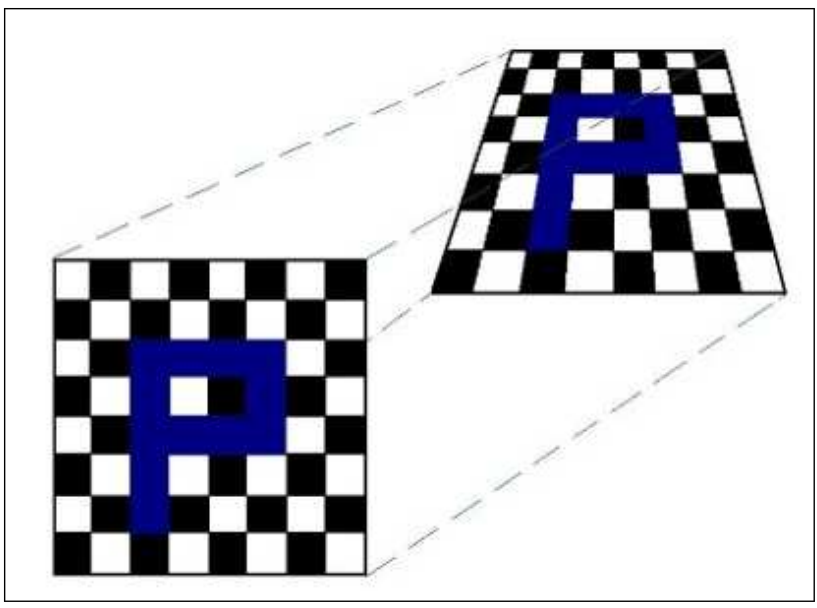

Figure 3: Image perspective 
to facilitate the calibration. The homography map points (equation 1) from the work plane $(z=0)$ to the points in the image plane (equation 2$)$. The homography components depend on the intrinsic camera parameters and its location in the space (extrinsic parameters), which are constants.

$$
\begin{gathered}
X=[X, Y, 1]^{T} \\
X^{\prime}=\left[X^{\prime}, Y^{\prime}, 1\right]^{T}
\end{gathered}
$$

The equation that represents the homography transformation is given by equation (3), where the homography matrix $\mathrm{H}$ can be obtained by using four points in the calibrated image and the image in perspective [6].

$$
\left|X^{\prime}\right|=|H||X|
$$

In the test bed, typical errors occur when two metallic plates to be welded are misaligned. The first step to correct the situation is to measure this misalignment using image processing tools. Once the positional misalignment is quantified, the information is sent to the robot controller for compensation. The methodology consists of using a laser beam (50 $\mathrm{mW}$ with $\lambda=656 \mathrm{~nm})$ aimed to both plates as it is shown in Figure 4.

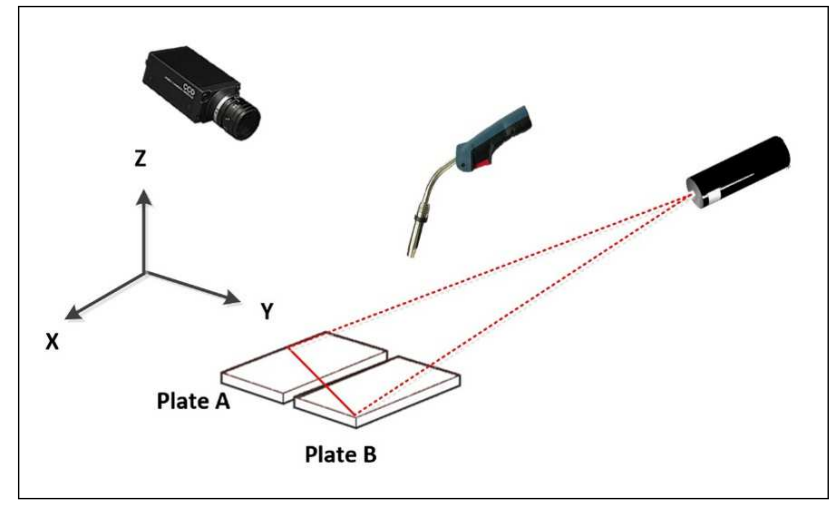

Figure 4: Welding methodology

When the laser beam is projected onto both plates, a discontinuous line appears and it is captured by the camera sensor as a reference signal. Misalignments can occur in two directions, either they can be lateral misalignments or height misalignments. In both cases, the misalignment is captured in the image. In order to accurately process this information, the image pre-processing is carried out first by smoothing the image applying a mean filter and $\max / \mathrm{min}$ filtering to reduce noise.

After the pre-processing stage, the image is segmented in the region of interest (ROI) where the necessary information is found (pixel $I_{i, j}$ in Figure 5).

The main idea is to take an image of dimension $K \mathrm{x} L$ from the original I image with dimension $I \mathrm{x} J$, so that $I(K \mathrm{x} L)<I(I \mathrm{x} J)$. Having a reduced image in size is also useful to speed up the processing time. The segmented ROI is shown in Figure 6. The gap between Plate A and Plate $\mathrm{B}$ can be observed within the rectangle formed by the dashed lines.

Once the gap is determined, the next step is to determine Discontinuity 1 and Discontinuity 2 from the laser pattern and captured from each plate as showed in Figure 7.

During normal welding operation, the robot's torch should be located in the middle point between the plates as it is indicated (Robot) in Figure 8. In the case of any offset either lateral 


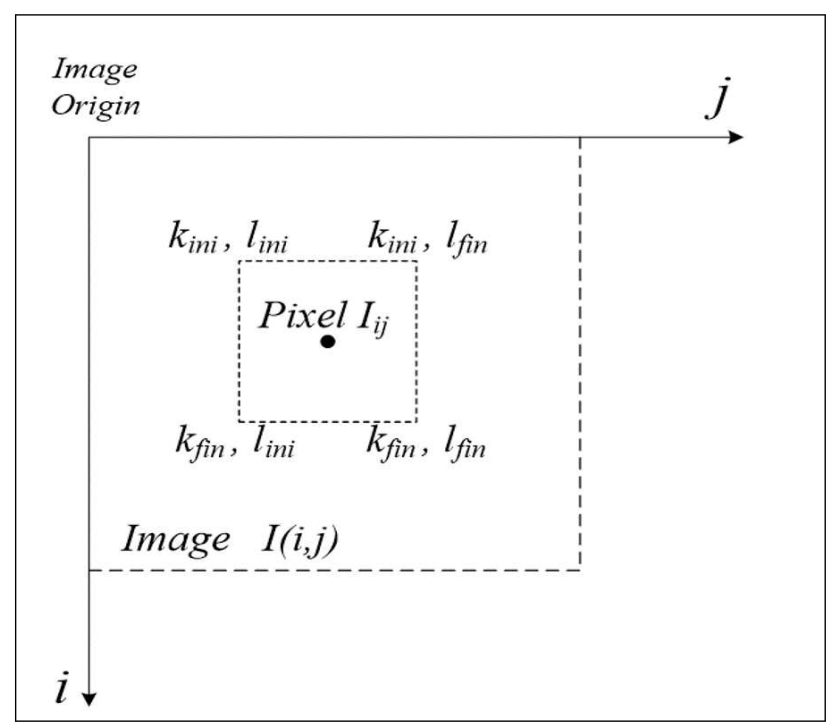

Figure 5: Image Segmentation

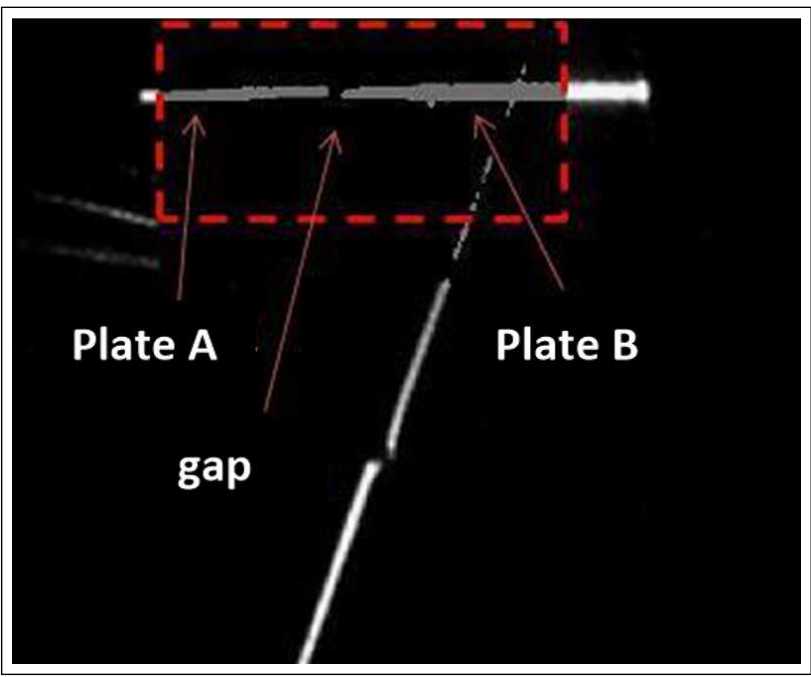

Figure 6: ROI with segmented image

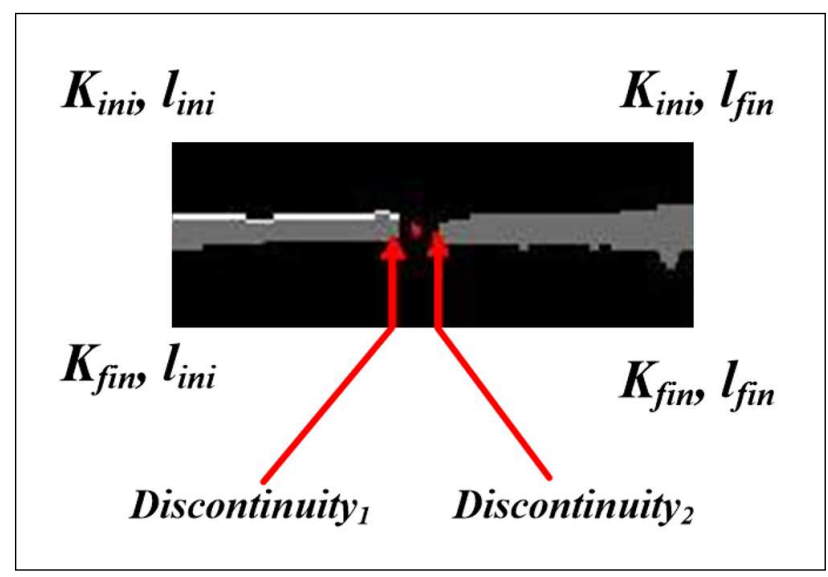

Figure 7: Discontinuity between plates 
misalignment or misalignment in the $Z$ axis direction, the point will move accordingly, hence requiring a repositioning strategy.

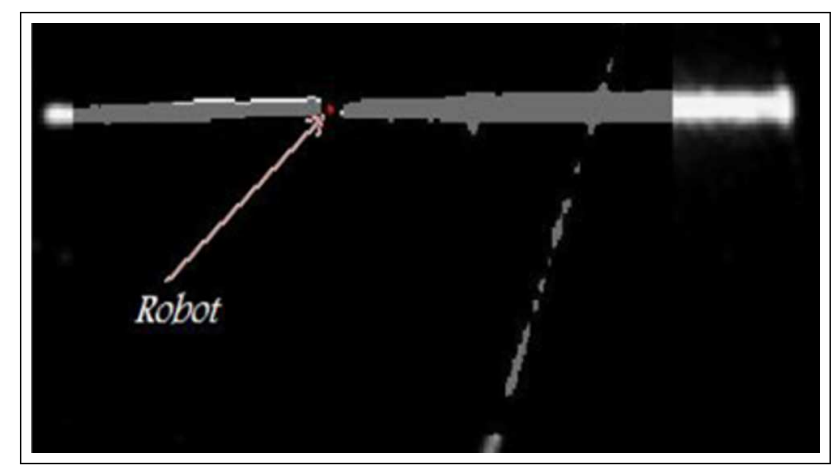

Figure 8: Point without offset position

In situations where the robot's torch is not in the correct position, the situation has to be assessed first by measuring the misalignment. A corrective motion will be a distance between points union $(x, y)$ and robot $(x, y)$ as depicted in Figure 9. The corrective motion has to be in any direction within 3D space volume, so that we proposed a Fuzzy algorithm to correct it.

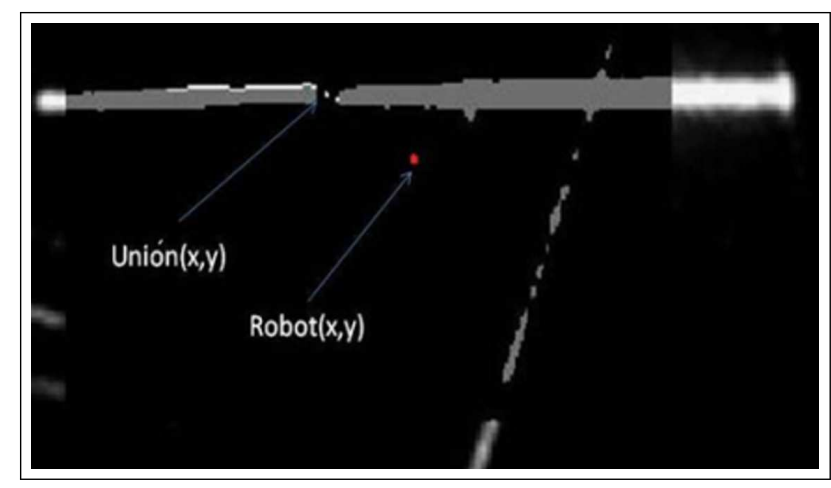

Figure 9: Torch offset position

\section{Design of the controller}

In our experiments two controllers were tested. The first controller is based on the Type-1 Fuzzy Logic Control which uses triangular and trapezoidal membership functions. The second controller uses Gaussian membership functions and is referred to as Singleton Fuzzy Logic Controller.

According to Mendel [7] membership functions characterize fuzzy sets. A type-1 (T1) fuzzy set, $A$ which is in terms of a single variable $x \in X$, is a generalization of a crisp set. It is defined on a universe of discourse $X$ and is characterized by membership function $\mu_{A}(x)$ that takes on values in the interval $[0,1]$. A membership function provides a measure of the degree of similarity of an element in $X$ to the fuzzy set. Such a set may be represented as

$$
A=\left\{\left(x, \mu_{A}(X)\right) \mid \forall x \in X\right\}
$$

$T-1$ membership function, $\mu_{A}(x)$ is constrained to be between 0 and 1 for all $x \in X$, a Gaussian membership function is specified by two parameters $m, \sigma$ as follows: 


$$
\operatorname{gausian}(x: m, \sigma)=\exp \left(-\frac{(x-m)^{2}}{\sigma^{2}}\right)
$$

where $m$ and $\sigma$ denote the center and width of the function, respectively. The shape of the function can be controlled by adjusting the parameter $\sigma$. A small value of $\sigma$ will generate "thin" membership function, while a big $\sigma$ will lead to a "flat" membership function.

Fuzzy logic controllers (FLC's) are useful control schemes for plants having difficulties in deriving mathematical models or having performance limitations with conventional control schemes. Error $e$ and change of error $e^{\prime}$ are the most used fuzzy input variables in most fuzzy control works, regardless of the complexity of controlled plants. Also, either control input ${ }^{u}(P D-$ type $)$ or incremental control input ${ }^{\Delta u}(P I-$ type $)$ is typically used as fuzzy output variable. T1 FLC's are both intuitive and numerical systems that map crisp inputs to a crisp output. Every FLC is associated with a set of rules with meaningful linguistic interpretations, obtained from either numerical data, or experts. Based on this kind of statement, actions are combined with rules in an antecedent-consequent format, and then aggregated according to approximate reasoning theory, to produce a nonlinear mapping from input space $X 1 \times X 2$ to the output space $Y$.

\subsection{Input-output relationship}

A fuzzy control in the general case is based on linguistic variables to handle imprecise and vague information. The information is embedded in fuzzy sets that are combined in rules to define actions to be taken as it is indicated in [8] and [9]. In our case, we need to define a set of input-output relationships in order to quantify the positional error of the robot arm with respect to the plate's welding position. In other words, the input information for the robot is the misalignment of the robot's end effector and the output is a robot position command to correct such misalignment.

The design of the tracking system is focused on the offset compensation in automatic production lines, where the parts to be welded are moved by pallets to other welding stations and where typical positioning errors are in the range of few millimetres. These errors are likely to occur between the current workpiece and the next due to several reasons as mentioned earlier. During these circumstances, the user would normally correct the robot path by reprogramming the robot which is time consuming. Our proposal is to carry out the correction on-line, using the same welding program. The idea is to modify incrementally the robot's offset path at the starting of the welding operation should a misalignment is detected so that not reprogramming is needed. In order to modify the path a deviation measurement has to be compared to the original path, so that the robot "knows" where to move to and for how much using linguistic variables such as "right", "left", "far left", "decrease slightly", "increase greatly", etc. The linguistic variables are translated into fuzzy sets with a membership function that considers a value in the range $[0,1]$.

\subsection{Fuzzy design}

The steps to be considered in the design are normalisation, fuzzification, determination of fuzzy rules, defuzzyfication, and denormalisation. The first step is accomplished by using the information given from a sensing system, which in this case is obtained from the laser beam pattern as detected by the camera. This value is read and must be normalised to the range $[0,1]$. The next step is to fuzzify this information to assess its membership within the fuzzy sets. In our case the variable depends on the workpiece misalignment and we should find its membership function within the following fuzzy sets: far left, left, near left, near, near right, right, and far right. 
Once the input data has been normalised and fuzzified, then this is used to build up the fuzzy rules what is meant to be the control antecedent. These rules are built depending on the requirement of the system and after its evaluation their membership function is determined in relation with the output set (consequent). In order to have useful singleton real values, the data set is defuzzified to obtain a new robot coordinate which is in turn sent to the robot controller to ultimately correct the welding trajectory.

If we consider the $X$ axis to be the welding direction as depicted in Figure 4, it is clear that the misalignment would come only in the $Y$ or $Z$ axis direction. Considering this assumption, the control system is based on two input variables and two output variables. The operation range is defined in the interval $[-10,10] \mathrm{mm}$ in the $Y$ axis and $[-5,5] \mathrm{mm}$ in the $Z$ axis. The zero value is considered to be the reference value for the welding path. Figure 10 shows a set of input data in the $Y$ and $Z$ axes during the evaluation tests of the controller. These values comprise the whole set of values likely to be encountered during operations. For instance, if the algorithm detects a misalignment point $(10,-5)$ it means that the workpiece had an offset of $10 \mathrm{~mm}$ to the right and $-5 \mathrm{~mm}$ downwards that needs to be compensated.

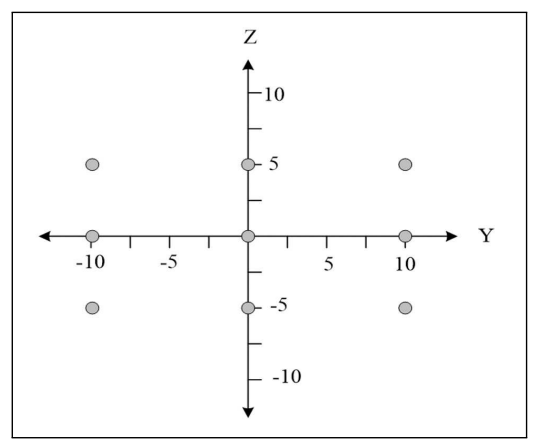

Figure 10: Robotic motion range for error compensation

The trapezoidal and triangular membership functions are used to design the input and output fuzzy sets as shown in the Figure 11.

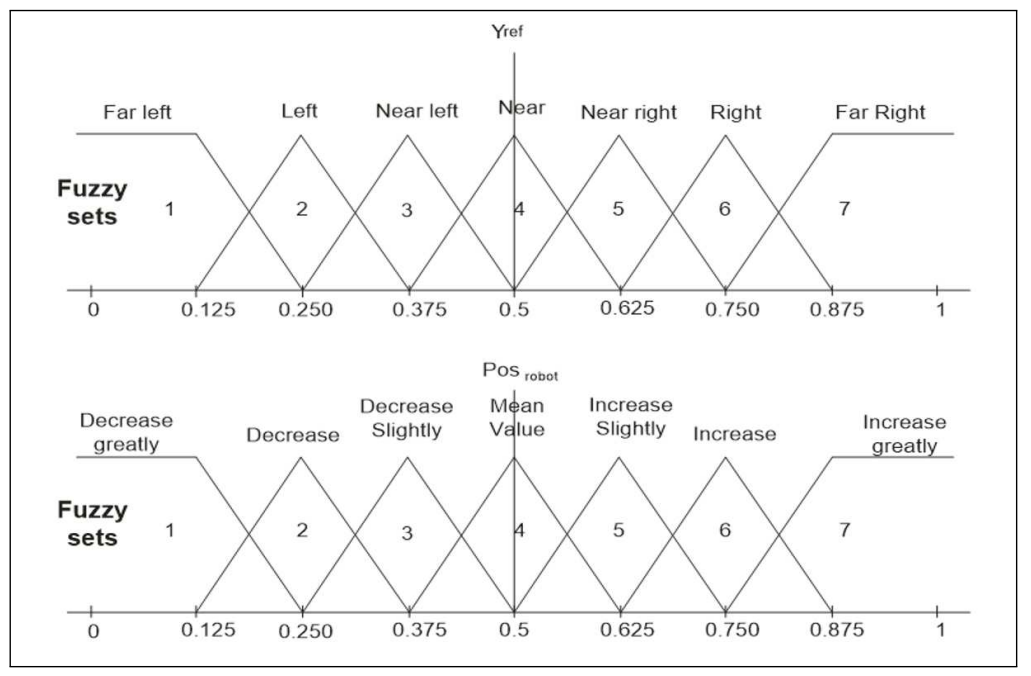

Figure 11: Fuzzy sets

After the degree of membership of the input values from the input fuzzy sets is defined, the antecedent is created using fuzzy rules as follows: 


$\begin{array}{ccccccc}\text { IF } & \text { Yref } & \text { IS } & \text { Far left } & \text { THEN } & \text { Decrease greatly } & \text { Posrobot } \\ \text { IF } & \text { Yref } & \text { IS } & \text { left } & \text { THEN } & \text { Decrease } & \text { Posrobot } \\ \text { IF } & \text { Yref } & \text { IS } & \text { Near left } & \text { THEN } & \text { Decrease slightly } & \text { Posrobot } \\ \text { Etc. } & \ldots & \ldots & \ldots & \ldots & \ldots & \ldots\end{array}$

During the defuzzyfication stage the required non-fuzzy real values are obtained using the Centre of Area (CoA) method as a fuzzy conclusion "Y is A". This can be determined by equation 6 in the discrete domain.

$$
y=\frac{\sum_{i} \mu_{A}\left(y_{i}\right) \times y_{i}}{\sum_{i} \mu_{A}\left(y_{i}\right)}
$$

where $\mu_{A}(y i)$ is the membership function.

Having defuzzified the data, their values are denormalised obtaining a real value which is used as a new robot coordinate. The fuzzy robot controller will also consider other errors related to other variables such as ageing of the positioning mechanisms or disturbances. The correction is on-line, during the welding process avoiding the need of stopping the production line. The operating working range is $\pm 10 \mathrm{~mm}$ in $\mathrm{Y}$ axis and $\pm 5 \mathrm{~mm}$ in $\mathrm{Z}$ axis. The fuzzy controller was developed in $\mathrm{C}++$ using the Visual Studio compiler.

\subsection{Performance assessment}

In order to assess the statistical performance of the controller a set of experiments were carried out. The robot path tracking ability during the operation range was evaluated $\pm 10 \mathrm{~mm}$ in $Y$ axis and $\pm 5 \mathrm{~mm}$ in $Z$ axis. The evaluation helps to analyse its behaviour against any variation of the experimental factors. The factors are considered either combined or in its individual form so that the interaction can be identified as indicated in [7]. Care was taken to consider two important aspects during the experimental design that are replicate and aleatorisation. The use of replicates is very important to determine the experimental error. The aleatorisation allows confirming that the random probability variables refer to independent probability distributions. Considering the above assumption, the input variables to the system are the position values that are sent to the robot in the $Y$ and $Z$ axis direction (DistY,Dist $Z$ ) and as output, the real distance observed in both axes (DistY real and DistZ real).

Experimental design considers variables with two or three levels and $k$ factors referred as to $2 K$ or $3 K$, respectively. In our experiments we decided to use 32 with 2 replicates. The decision to use three levels was based on the interest of using the central point within the robot's range motion in both, the $Y$ axis and the $Z$ axis.

\section{Results}

\subsection{Results with the T1 FLC}

The experimental procedure can be observed in Figure 12. The experimental set up and the coordinate frame are shown. The offset value (DistY, DistZ) that the fuzzy controller has to compensate is showed in the dashed rectangle. The output value is considered as the real value measured along the main axes (DistY real, DistZ real). It is important to note that the welding seam is applied along the $X$ axis.

Table 1 contains the results from 18 experimental runs. The first 9 corresponds to the first replicate and runs 10 to 18 correspond to the second replicate. Figure 13 shows results obtained during the correction of lateral misalignment and the measured absolute error. Similarly, Figure 14 shows the obtained results during the correction in the vertical distance and its absolute 


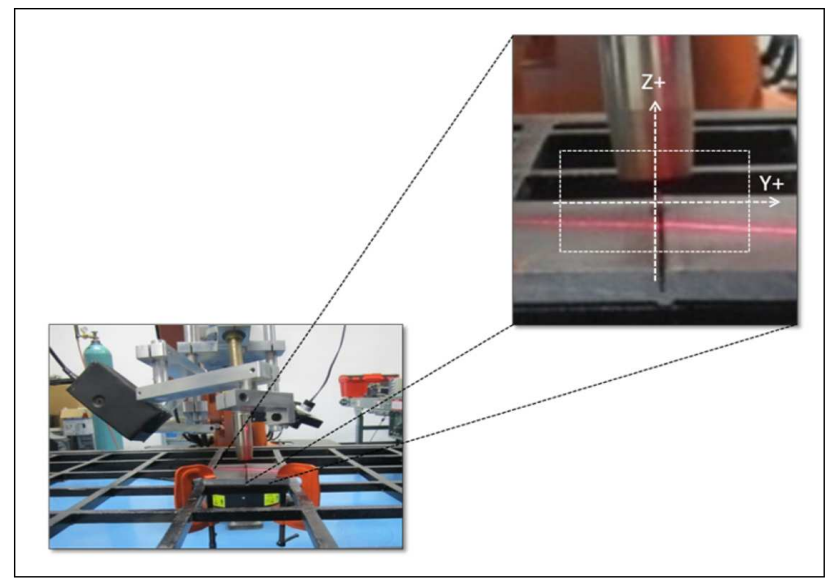

Figure 12: Experimental set up

error. The measured error demonstrates that the robot fuzzy controller compensates the misalignment in all cases. The maximum observed error is $1.6 \mathrm{~mm}$ in $Y$ axis, which is considered to be appropriate for practical purposes in industrial manufacturing processes.

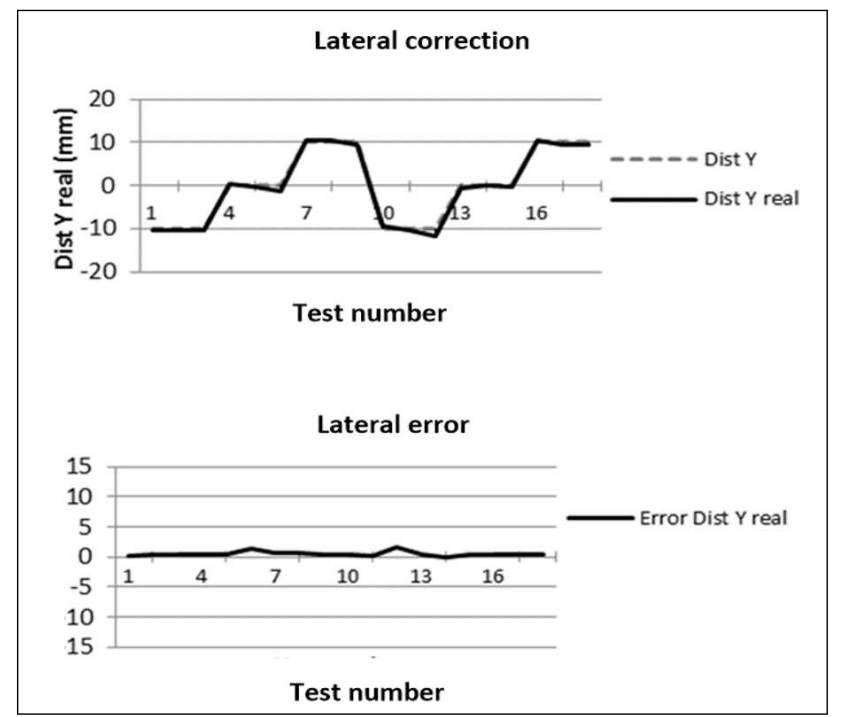

Figure 13: Lateral misalignment and absolute error

In comparison with the triangular T1 FLC, the Gaussian T1 FLC uses a bigger area of exploration. It was decided to duplicate the area $40 \mathrm{~mm} \times 20 \mathrm{~mm}$. The lateral misalignment range is $+/-20 \mathrm{~mm}$ in the $\mathrm{Y}$ axis whereas the vertical misalignment is $+/-10 \mathrm{~mm}$ in the $\mathrm{Z}$ axis. This can be observed in Figure 15.

The Gaussian T1 FLC uses more information as it considers mid points between the initial and final points. Every point within the exploration area forms a fuzzy rule. If we consider that the points are spaced $2 \mathrm{~mm}$ then we will have 21 points in $\mathrm{Y}$ axis by 11 in the $\mathrm{Z}$ axis making a total of 231 points or fuzzy sets for the Singleton FLC. The Gaussian type of fuzzy sets are illustrated in Figure 16.

The Gaussian controller has the advantage of not requiring the homography since the input information is given directly from the camera in pixels. For the reference point $(0,0)$ which is supposed to be the point where the welding torch should be aligned with is located in the point 
Table 1: Experimental results and error evaluation ( $\mathrm{mm})$

\begin{tabular}{cccccccc}
\hline DistY & DistY Real & Error Y & DistZ & DistZ Real & Error Z & AbsY error & AbsZ error \\
\hline-10 & $-10,2$ & 0.2 & -5 & -5 & 0 & 0.2 & 0 \\
-10 & -10.4 & 0.4 & 0 & -0.4 & 0.4 & 0.4 & 0.4 \\
-10 & -10.4 & 0.4 & 5 & 4.4 & 0.6 & 0.4 & 0.6 \\
0 & 0.47 & -0.47 & -5 & -5.26 & 0.26 & 0.47 & 0.26 \\
0 & -0.43 & 0.43 & 0 & -0.42 & 0.42 & 0.43 & 0.42 \\
0 & -1.3 & 1.3 & 5 & 5.28 & -0.28 & 1.3 & 0.28 \\
10 & 10.6 & -0.6 & -5 & -5.2 & 0.2 & 0.6 & 0.2 \\
10 & 10.6 & -0.6 & 0 & 0.6 & -0.6 & 0.6 & 0.6 \\
10 & 9.6 & 0.4 & 5 & 5.2 & -0.2 & 0.4 & 0.2 \\
\hline-10 & -9.5 & -0.5 & -5 & -4.95 & -0.05 & 0.5 & 0.05 \\
-10 & -10.2 & 0.2 & 0 & -0.2 & 0.2 & 0.2 & 0.2 \\
-10 & -11.6 & 1.6 & 5 & 4.9 & 0.1 & 1.6 & 0.1 \\
0 & -0.53 & 0.53 & -5 & -5.3 & 0.3 & 0.56 & 0.3 \\
0 & 0 & 0 & 0 & -0.1 & 0.1 & 0 & 0.1 \\
0 & -0.33 & 0.33 & 5 & 5.27 & -0.27 & 0.33 & 0.27 \\
10 & 10.5 & -0.5 & -5 & -5 & 0 & 0.5 & 0 \\
10 & 9.6 & 0.4 & 0 & -0.4 & 0.4 & 0.4 & 0.4 \\
10 & 9.6 & 0.4 & 5 & 5 & 0 & 0.4 & 0 \\
\hline
\end{tabular}

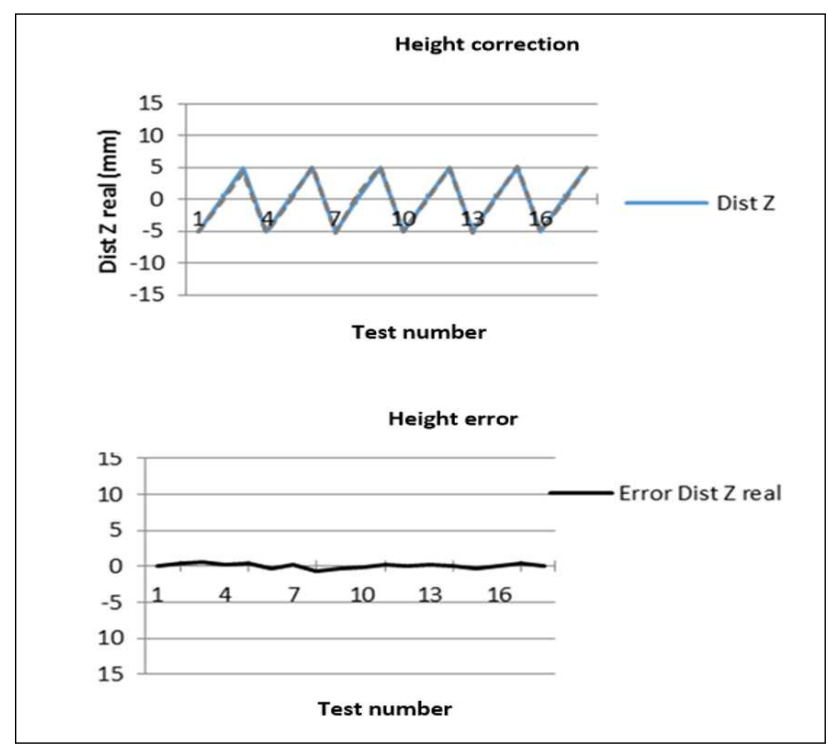

Figure 14: Vertical misalignment and absolute error 


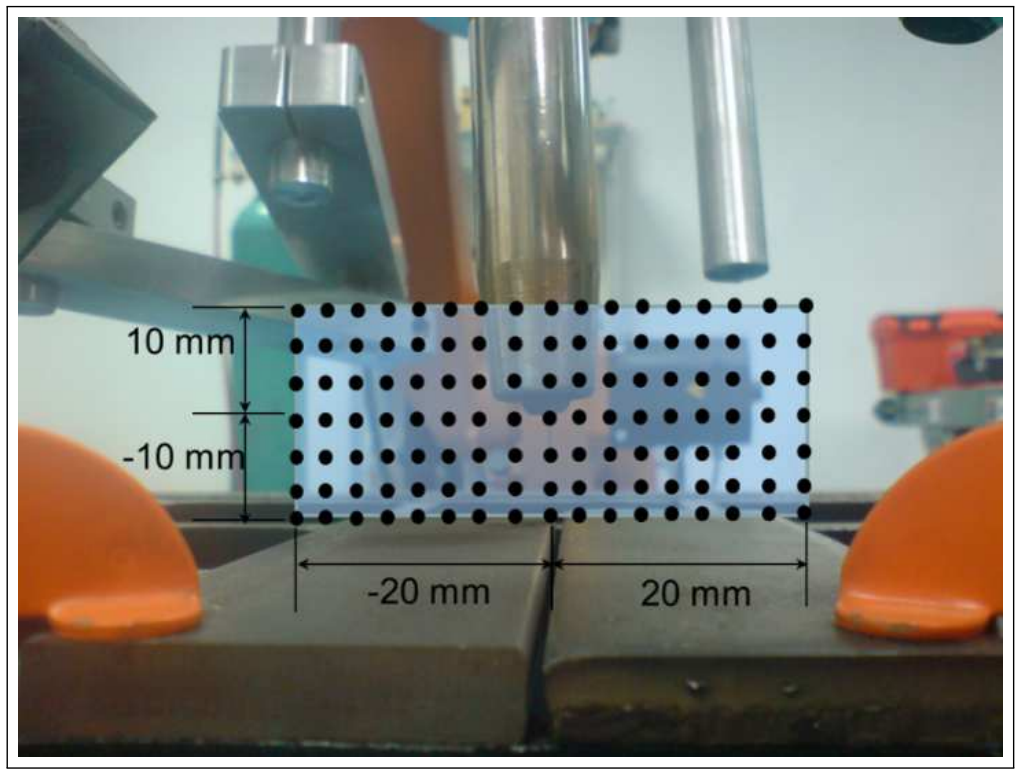

Figure 15: Exploration area in the $Y$ and $Z$ axis

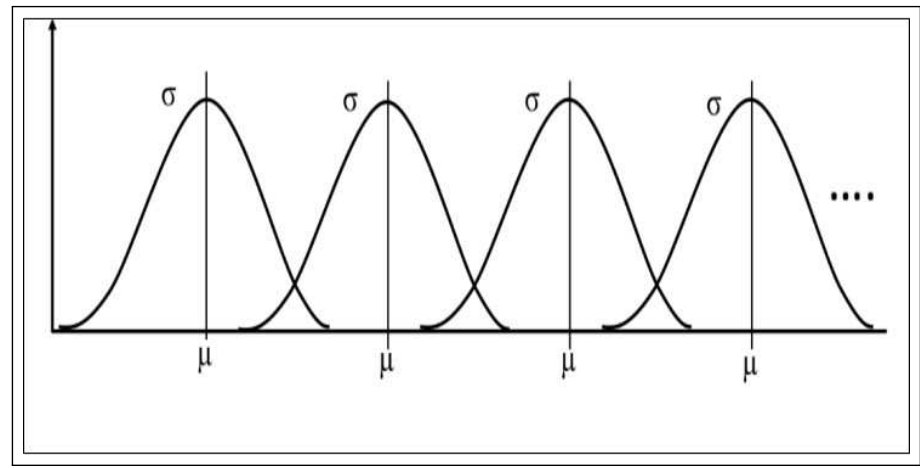

Figure 16: Gaussian type fuzzy sets 
Table 2: Results from the Singleton FLC

\begin{tabular}{|c|c|c|c|c|c|c|}
\hline No. & Y axis & Z axis & PosRobot $_{Y}$ & PosRobot $_{Z}$ & Error Y axis & Error Z axis \\
\hline 1 & -20 & 10 & 19.59255 & -9.99768 & 0.40745 & 0.00232 \\
2 & -20 & 0 & 19.98974 & 0.87296 & 0.01026 & 0.87296 \\
3 & -20 & -10 & 19.8391 & 10 & 0.1609 & 0 \\
4 & 0 & 10 & 0.02595 & -10 & 0.02595 & 0 \\
5 & 0 & 0 & -0.02274 & 0.00005 & 0.02274 & 0.00005 \\
6 & 0 & -10 & 0.02708 & 9.99652 & 0.02708 & 0.00348 \\
7 & 20 & 10 & -19.90655 & -10 & 0.09345 & 0 \\
8 & 20 & 0 & -19.96467 & 0.0156 & 0.03533 & 0.0156 \\
9 & 20 & -10 & -19.99986 & 10 & 0.00014 & 0 \\
10 & -19 & 9 & 19.79273 & -9.99535 & 0.79273 & 0.99535 \\
11 & -19 & 10 & 17.99499 & -10.04568 & 1.00501 & 0.04568 \\
12 & -17 & 10 & 18.09578 & -10 & 1.09578 & 0 \\
13 & -10 & 5 & 9.16485 & -5.54219 & 0.83515 & 0.54219 \\
14 & -10 & 0 & 10.45749 & -0.00265 & 0.45749 & 0.00265 \\
15 & -10 & -5 & 10.87992 & 4.61136 & 0.87992 & 0.38864 \\
16 & 0 & 5 & 0.197392 & -5.95809 & 0.197392 & 0.95809 \\
17 & 0 & 0 & -0.0322 & 0.05833 & 0.0322 & 0.05833 \\
18 & 0 & -5 & 0.07501 & 5.80521 & 0.07501 & 0.80521 \\
19 & 10 & 5 & -10.03783 & -5.96332 & 0.03783 & 0.96332 \\
20 & 10 & 0 & -9.96102 & 0 & 0.03898 & 0 \\
21 & 10 & -5 & -9.15139 & 4.04127 & 0.84861 & 0.95873 \\
\hline
\end{tabular}

$(3.34598,-3.07248)$ pixels and so on with the other 230 points located within the total area. Once all points are related within the image, then the next step is to form the fuzzy sets by using the Gaussian function given by equation (5).

Since the information comes from the process and does not require normalisation, the number of fuzzy sets are 231 and its membership will be given by the mean and standard deviation from each point. In order to generate the fuzzy sets it is required to carry out at least 10 tests in each point, so the total number of tests are 2,310 points to generate the fuzzy sets.

To validate the performance of the Gaussian T1 FLC several tests were carried out within the exploration area. The position error of the torch in the $\mathrm{Y}$ and $\mathrm{Z}$ axis are the input variables (given in pixels), while the output variables are the robot's end effector coordinates given by PosRobot $_{Y}$ and PosRobot $_{Z}$. The Table 2 shows the results obtained after 21 trials. It can be observed that errors are lower compared to the case of the triangular T1 FLC and having a maximum value of approximately $1 \mathrm{~mm}$.

Figures 17 and 18 show the corresponding error graphs where it is clear that the misalignment of the plates was corrected and in all cases the repositioning of the arm had an error lower than $1 \mathrm{~mm}$, which is better that in the case of the triangular T1 FLC [1].

\section{Conclusions}

This article presented an alternative solution to this problem that involves the use of structured lighting using a low-cost laser beam, a CMOS camera and a singleton type-1 fuzzy logic controller. To validate the proposed control system, a robotic cell was designed using an industrial KUKA KR16 robot for welding metallic plates. The method was evaluated experimentally 


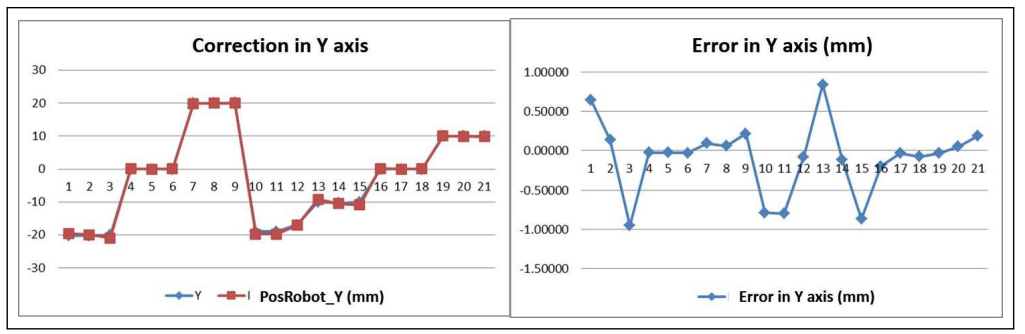

Figure 17: Relationship between offset error and correction in the $\mathrm{Y}$ axis

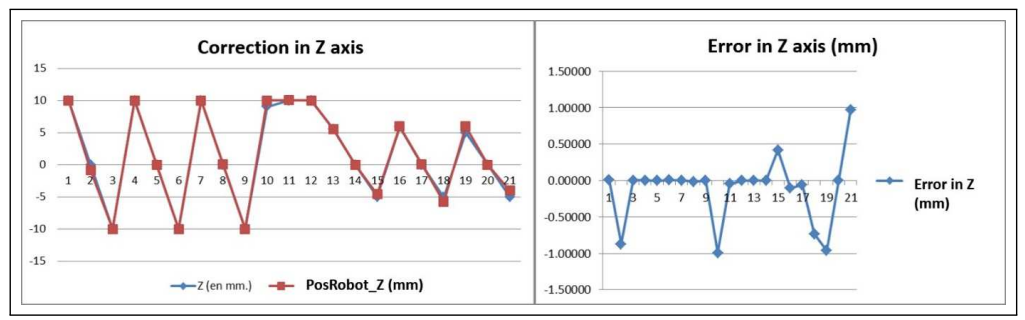

Figure 18: Relationship between offset error and correction in the $\mathrm{Z}$ axis

under lateral and vertical positioning errors. The methodology presented is for the correction of welding trajectories due to positioning errors using structured lighting and Gaussian T1 FLC.

The implementation results demonstrated the feasibility of the proposed Gaussian T1 FLC. The controller was validated experimentally with errors within the working range $\pm 20 \mathrm{~mm}$ for the $\mathrm{Y}$ axis and $\pm 10 \mathrm{~mm}$ in the $\mathrm{Z}$ axis. During experiments, the obtained results showed a maximum error of $1 \mathrm{~mm}$, which is considered appropriate in practical manufacturing tasks.

The statistical analysis of the results indicated that there is statistical evidence to consider a mean of zero value for average error in both axes with $\alpha=0.01$ for the triangular T1 FLC, while it shows that the Gaussian T1 FLC is more efficient since it works under a type system exploring the area more exhaustive using intermediate points. In addition, due to a greater number of diffuse rules and the use of Gaussian functions that include the value of the media and of the deviation standard, the model includes the uncertainties (noise) of the process sensors, i.e., the number of pixels detected during the processing of the image, which vary with the different levels of illumination.

Future work is looking into two areas: inspection of welding seam and type-2 fuzzy logic controllers. In terms of inspection is intended to expand the scope of the project by including the verification of width and height of the seam using a camera with greater dynamic range (approximately $120 \mathrm{~dB}$ ), and the use of logarithmic algorithms that reduce high luminance that are present during the welding process.

\section{Acknowledgment}

Authors want to acknowledge the Consejo Nacional de Ciencia y Tecnología (CONACyT) for the scholarship provided to Ignacio Dávila-Ríos during his $\mathrm{PhD}$ studies, as well as the grant support from CONACyT No. 61373. 


\section{Bibliography}

[1] Ignacio Davila-Rios, Ismael Lopez-Juarez, Gerardo M. Mendez, Roman Osorio-Comparan, Gaston Lefranc and Claudio Cubillos (2016); A Fuzzy Approach for on-line Error Compensation During Robotic Welding, Computers Communications and Control (ICCCC), 2016 6th International Conference on, ISBN 978-1-5090-1735-5, IEEE Xplore, DOI:10.1109/ICCCC.2016.7496766, 264- 270.

[2] Menno de Graaf, Ronald Aarts, Ben Jonker, Johan Meijer (2010); Real-time seam tracking for robotic laser welding using trajectory-based control, Control Engineering Practice, 18(8):944-953.

[3] Tero Santti, Jonne K. Poikonen, Olli Lahdenoja, Mika Laiho, Ari Paasio (2015); Online seam tracking for laser welding with a vision chip and FPGA enabled camera system, IEEE International Symposium on Circuits and Systems (ISCAS), May 2015, 1985 - 1988.

[4] Jinchao Liu, Zhun Fan, Srrren Ingvor Olsen, Kim Hardam Christensen, Jens Klćstrup Kristensen (2015); A Real-time Passive Vision System for Robotic Arc Welding, IEEE International Conference on Automation Science and Engineering (CASE), Aug. 2015, 389-394.

[5] T. Ngo, Y. Wang, T.L. Mai, M.H. Nguyen, J. Chen (2012); Robust Adaptive Neural-Fuzzy Network Tracking Control for Robot Manipulator, International Journal of Computer Communications $\& 3$ Control, ISSN 1841-9836, 7(2):341-352.

[6] I. Hartley and A. Zisserman (2004); Multiple View Geometry in Computer Vision, Cambridge University Press, ISBN: 0521540518, second edition, 2004.

[7] J. M. Mendel (2001); Uncertain rule-based fuzzy logic systems: introduction and new directions, Prentice-Hall, Upper Saddle River, NJ, 2001.

[8] John Yen, Reza Langari (1998); Fuzzy Logic: Intelligence, Control and Information, PrenticeHall), ISBN 0135258170, 1998.

[9] Martín del Br io B, Sanz Molina A. (2006); Redes Neuronales y Sistemas Borrosos, 3rd edition, ISBN 978-84-7897-743-7, RA-MA, 2006.

[10] Montgomery D C. (2007); Design and Analysis of Experiments, John Wiley Sons, 6th edition, ISBN-10: 047148735X, 2007. 